\title{
Hardy Relations and Common Cause
}

\author{
Katsuaki Higashi
}

April 25, 2020

\begin{abstract}
Some researchers argued that in the non-existence proof of hidden variables, the existence of a common common-cause of multiple correlations is tacitly assumed and that the assumption is unreasonably strong. According to their idea, it is sufficient if the separate common-cause of each correlation exists. However, for such an idea, various no-go results are already known. Recently, Higashi showed that there exists no local separate common-cause model for the correlations that appear in Hardy's famous argument. In this paper, I give another simple and suggestive proof of the same content. First, I will show that there exists no local common common-cause model of the correlations that appear in Hardy's argument. Second, taking the proof as a hint, following almost the same steps, I will show the non-existence of a local separate common-cause model for those correlations. Finally, based on the argument in the previous sections, I will discuss what we can conclude about the issue of reducibility from a separate common-cause model to a common common-cause model. It will be concluded that it is "irreducible" at least by a usual method.
\end{abstract}

\section{Introduction}

The conventional view is that there exists no common cause of quantum correlations (e.g. $[1,2])$. Because, when we assume the existence of a common cause, Bell's inequalities are derived and the violation of the inequalities has been repeatedly confirmed by the experiments (e.g. [3]). On the other hand, some researchers $[4,5,6]$ argued that in the derivations of Bell's inequalities, it is tacitly assumed that there exists a common common-cause in multiple correlations and the assumption is a too strong requirement. They consider what is needed to explain the quantum mechanical correlation is a separate common-cause for each correlation. Although this idea is very interesting, various no-go theorems for separate common-cause approaches are already known $[7,8,9,10,11,12,13,14]$.

Graßhoff et al. [7] showed that there exists no local separate common-cause model in the maximally entangled states. In addition, Higashi [14] recently used Hardy's famous argument to show that, in any non-maximally entangled state, there exists no local separate common-cause model. Therefore, it became clear 
that, in any entangled state, there exists no local separate common-cause model. In this paper, I present another simple and suggestive proof of the mathematical fact which Higashi proved (see Fact 6 in [14]).

First, in Sect. 3.1, I will show that there exists no local common commoncause model for multiple correlations that appear in Hardy's argument. After that, in Sect. 3.2, I will show that there exists no local separate common-cause model for those correlations in a similar way to in Sect. 3.1. By comparing these two proofs, it will be clear how effective (or non-effective) the strategy to weaken the requirement for a common common-cause to the one for a separate common-cause for each correlation. Finally, in Sect. 4, I will discuss whether non-existence proof of the separate common-cause is reduced to non-existence proof of the common common-cause, based on the analysis in Sect. 3. This is the issue raised and discussed by Hofer-Szabó $[10,11,12]$ and recently discussed by Wroński et al. [13].

\section{Background}

\subsection{Hardy Relations}

Let $\mathcal{H}_{L} \otimes \mathcal{H}_{R}$ be a tensor product Hilbert space composed of two 2-dimensional Hilbert spaces $\mathcal{H}_{L}$ and $\mathcal{H}_{R}$ of the left and right systems, respectively. For any pure state in $\mathcal{H}_{L} \otimes \mathcal{H}_{R}$, the following mathematical fact holds (for proof, see e.g. [15]).

Schmidt decomposition For any pure state $\phi$ in $\mathcal{H}_{L} \otimes \mathcal{H}_{R}$, there exists an orthonormal basis $\left\{e_{1}, e_{2}\right\}$ for $\mathcal{H}_{L}$, and an orthonormal basis $\left\{f_{1}, f_{2}\right\}$ for $\mathcal{H}_{R}$ such that

$$
\phi=\alpha e_{1} \otimes f_{1}+\beta e_{2} \otimes f_{2},
$$

where $\alpha, \beta$ are non-negative real numbers satisfying $\alpha+\beta=1$ known as Schmidt coefficients.

Schmidt decomposition is unique (ignoring the phase factor) if and only if all Schmidt coefficients are distinct (i.e. $\alpha \neq \beta$ in 2-dimensional case). When more than one Schmidt coefficients of a state are non-zero (i.e. $\alpha, \beta \neq 0$ ), we call the state an entangled state. In particular, when all Schmidt coefficients are identical (i.e. $\alpha=\beta$ ), it is called a maximally entangled state (e.g. the singlet state). We call entangled states other than maximally entangled states non-maximally entangled states. Hardy [16] showed that for any non-maximally entangled state $\psi$ in $\mathcal{H}_{L} \otimes \mathcal{H}_{R}$, there exist projection operators $A_{L}, B_{L}$ on $\mathcal{H}_{L}$ and $A_{R}, B_{R}$ on $\mathcal{H}_{R}$ that satisfy the following four relations A1-4. (Below, we abbreviate the projections $A_{L} \otimes I, B_{L} \otimes I, I \otimes A_{R}, I \otimes B_{R}$ on $\mathcal{H}_{L} \otimes \mathcal{H}_{R}$ as $A_{L}, B_{L}, A_{R}, B_{R}$, respectively.)

A1 $Q^{\psi}\left(A_{L} A_{R}\right)>0$,

A2 $Q^{\psi}\left(B_{R} \mid A_{L}\right)=1$ 
A3 $Q^{\psi}\left(B_{L} \mid A_{R}\right)=1$,

A4 $Q^{\psi}\left(B_{L} B_{R}\right)=0$,

where $Q^{\psi}(\cdot)$ represents the quantum mechanical probability in a state $\psi$. We call these four relations Hardy relations.

Hardy argued that there exists no truth-value assignment that satisfies A1 to A4. His argument goes as follows. Suppose $A_{L} \wedge A_{R}$ is true (this is possible by A1). Then, using $\mathrm{A} 2$ and $\mathrm{A} 3, B_{L} \wedge B_{R}$ is true. However, this contradicts with A4.

If the four events corresponding respectively to the four projections $A_{L}, B_{L}$, $A_{R}, B_{R}$ exist in the Boolean algebra of a classic probability space, and the quantum mechanical probabilities $Q^{\psi}(\cdot)$ are assigned to those events respectively, then Hardy's argument holds. However, when the quantum mechanical probabilities are regarded as conditional probabilities given the setting of the measuring device, it is known that those probabilities can be expressed in the classical probability space $[17,18]$. To express the quantum probabilities from A1 to A4 as such conditional probabilities, we introduce the following notations.

$L_{1}$ : the apparatus $\mathrm{L}$ is set to measure $A_{L}$.

$L_{1+}$ : the result of $A_{L}$-measurement is +1 .

$L_{2}$ : the apparatus $\mathrm{L}$ is set to measure $B_{L}$.

$L_{2+}$ : the result of $B_{L}$-measurement is +1 .

$R_{1}$ : the apparatus $\mathrm{R}$ is set to measure $A_{R}$.

$R_{1+}$ : the result of $A_{R}$-measurement is +1 .

$R_{2}$ : the apparatus $\mathrm{L}$ is set to measure $B_{R}$.

$R_{2+}$ : the result of $B_{R}$-measurement is +1 .

Then, Hardy relations A1-A4 are rewritten as follows:

B1 $\operatorname{Pr}\left(L_{1+} R_{1+} \mid L_{1} R_{1}\right)>0$,

B2 $\operatorname{Pr}\left(R_{2+} \mid L_{1} R_{2} L_{1+}\right)=1$,

B3 $\operatorname{Pr}\left(L_{2+} \mid L_{2} R_{1} R_{1+}\right)=1$,

B4 $\operatorname{Pr}\left(L_{2+} R_{2+} \mid L_{2} R_{2}\right)=0$.

Where to simplify the notation, " $\wedge$ " is omitted. (If not omitted, for example, B1 is $\operatorname{Pr}\left(L_{1+} \wedge R_{1+} \mid L_{1} \wedge R_{1}\right)$.) Similarly, omit " $\wedge$ " in the following.

In this way when the quantum probability is regarded as a conditional probability, the quantum mechanical correlation is expressed in the following form:

$\operatorname{Corr}\left(L_{i+}, R_{j+}\right) \equiv \operatorname{Pr}\left(L_{i+} R_{j+} \mid L_{i} R_{j}\right)-\operatorname{Pr}\left(L_{i+} \mid L_{i} R_{j}\right) \cdot \operatorname{Pr}\left(R_{j+} \mid L_{i} R_{j}\right) \neq 0$.

Also hereinafter, when not only there exists a correlation, but also $\operatorname{Pr}\left(L_{i+} \mid\right.$ $\left.R_{j+} L_{i} R_{j}\right)=1(0)$ and $\operatorname{Pr}\left(R_{j+} \mid L_{i+} L_{i} R_{j}\right)=1(0)$ hold, the correlation is called perfect (anti-)correlation. 
In this paper, we discuss only the common causal explanation of the correlations $\operatorname{Corr}\left(L_{1+}, R_{2+}\right)$ and $\operatorname{Corr}\left(L_{2+}, R_{1+}\right)$ in Hardy relations. Because it is sufficient to consider these two correlations to derive the contradiction with Hardy relations.

It may not be immediately obvious that $L_{1+}$ and $R_{2+}$ are correlated (i.e. $\operatorname{Corr}\left(L_{1+}, R_{2+}\right) \neq 0$ ) even if Hardy relations hold. Indeed, we cannot derive $\operatorname{Corr}\left(L_{1+}, R_{2+}\right) \neq 0$ from A2 alone. However, as will be confirmed below, when A2-4 hold, we get $\operatorname{Corr}\left(L_{1+}, R_{2+}\right) \neq 0$. Suppose that A2 and $\operatorname{Corr}\left(L_{1+}, R_{2+}\right)=$ 0 hold in an entangled state $\phi$ in $\mathcal{H}_{L} \otimes \mathcal{H}_{R} .{ }^{1}$ Then, $Q^{\phi}\left(B_{R} A_{L}\right)=Q^{\phi}\left(A_{L}\right)$ and $Q^{\phi}\left(B_{R} A_{L}\right)=Q^{\phi}\left(B_{R}\right) \cdot Q^{\phi}\left(A_{L}\right)$ hold and so we get $Q^{\phi}\left(B_{R}\right)=1$. Because $\phi$ is the entangled state in a tensor product Hilbert space composed of two 2dimensional Hilbert spaces, $B_{R}$ is the identity operator on $\mathcal{H}_{R}$. Then, from A4, we get $Q^{\phi}\left(B_{L}\right)=0$ however, this is incompatible with A3.

\subsection{Screening-off Factors of Correlation}

Suppose there exists a correlation between two events $E$ and $F$ (i.e. $\operatorname{Pr}(E F) \neq$ $\operatorname{Pr}(E) \cdot \operatorname{Pr}(F))$. If there is no direct causal relation between those two events, that is, when one event is not the cause of the other event, it is natural to think that a common cause $C$ of the correlated events exists and satisfies the following: ${ }^{2}$

- $\operatorname{Pr}(E F \mid C)=\operatorname{Pr}(E \mid C) \cdot \operatorname{Pr}(F \mid C)$,

- $\operatorname{Pr}\left(E F \mid C^{\perp}\right)=\operatorname{Pr}\left(E \mid C^{\perp}\right) \cdot \operatorname{Pr}\left(F \mid C^{\perp}\right)$.

Here, the reason for requiring not only the first condition but also the second condition is that if it is not satisfied, it is necessary to explain the correlation conditional on $C^{\perp}$.

In the above explanation, we considered $\left\{C, C^{\perp}\right\}$ as a set of common-causal events, however, there is no reason to restrict a set of events that explain the correlation only to such a set of two events. If there exists a logical partition that satisfies the next relation

$$
\operatorname{Pr}\left(E F \mid C_{i}\right)=\operatorname{Pr}\left(E \mid C_{i}\right) \cdot \operatorname{Pr}\left(F \mid C_{i}\right),
$$

those events would be candidates for a cause of the correlation. Therefore, we call the candidate for the cause of quantum correlation $\operatorname{Corr}\left(L_{i a}, R_{j b}\right) \neq 0$ the screening-off factors and define it as follows.

Definition 1. Suppose $L_{i a}$ and $R_{j b}$ are correlated (i.e. $\operatorname{Corr}\left(L_{i a}, R_{j b}\right) \neq 0$ ). We call the events $\left\{C_{k}^{i j}\right\}_{k \in K_{i j}}$ which satisfy the following conditions screeningoff factors of $\operatorname{Corr}\left(L_{i a}, R_{j b}\right)$.

(a) If $l \neq m\left(l, m \in K_{i j}\right)$, then $C_{l}^{i j} \wedge C_{m}^{i j}=\emptyset$.

\footnotetext{
${ }^{1}$ It is not necessary to limit to the non-maximally entangled state.

${ }^{2}$ This idea of requiring a common cause originated from Reichenbach [19].
} 
(b) $\operatorname{Pr}\left(C_{k}^{i j}\right) \neq 0$ for any $k \in K_{i j}$.

(c) $\sum_{k \in K_{i j}} \operatorname{Pr}\left(C_{k}^{i j}\right)=1$.

(d) $\operatorname{Pr}\left(L_{i a} R_{j b} \mid L_{i} R_{j} C_{k}^{i j}\right)=\operatorname{Pr}\left(L_{i a} \mid L_{i} R_{j} C_{k}^{i j}\right) \cdot \operatorname{Pr}\left(R_{j b} \mid L_{i} R_{j} C_{k}^{i j}\right)$.

In the following, for simplicity of discussion, I assume an index set of screeningoff factors is finite.

Note the following about this definition. In the notation of the screening-off factors $\left\{C_{k}^{i j}\right\}_{k \in K_{i j}}$ of the correlation $\operatorname{Corr}\left(L_{i a}, R_{j b}\right), a$ and $b$ do not appear. As can be easily ascertained, if there exist screening-off factors of one correlation (e.g. $\operatorname{Corr}\left(L_{i+}, R_{j+}\right)$ ), they are also screening-off factors of the other correlations $\left(\operatorname{Corr}\left(L_{i+}, R_{j-}\right), \operatorname{Corr}\left(L_{i-}, R_{j+}\right)\right.$, and $\left.\operatorname{Corr}\left(L_{i-}, R_{j-}\right)\right)$. Therefore, we do not need to specify a value of $a$ and $b$ in the notation of the screening-off factors.

Suppose that for events $X, Y$, and $Z, \operatorname{Pr}(X Y \mid Z)=\operatorname{Pr}(X \mid Z) \cdot \operatorname{Pr}(Y \mid Z)$. Then, as easily verified, if $\operatorname{Pr}(Y Z) \neq 0$, then $\operatorname{Pr}(X \mid Y Z)=\operatorname{Pr}(X \mid Z)$. Therefore, the following fact holds for screening-off factors.

Fact 1. When the events $\left\{C_{k}^{i j}\right\}_{k \in K_{i j}}$ are screening-off factors of the correlation $\operatorname{Corr}\left(L_{i a}, R_{j b}\right) \neq 0$,

(i) if $\operatorname{Pr}\left(L_{i} R_{j} L_{i a} C_{k}^{i j}\right) \neq 0$, then $\operatorname{Pr}\left(R_{j b} \mid L_{i} R_{j} L_{i a} C_{k}^{i j}\right)=\operatorname{Pr}\left(R_{j b} \mid L_{i} R_{j} C_{k}^{i j}\right)$.

(ii) if $\operatorname{Pr}\left(L_{i} R_{j} R_{j b} C_{k}^{i j}\right) \neq 0$, then $\operatorname{Pr}\left(L_{i a} \mid L_{i} R_{j} R_{j b} C_{k}^{i j}\right)=\operatorname{Pr}\left(L_{i a} \mid L_{i} R_{j} C_{k}^{i j}\right)$.

\section{On Common Cause Model of Hardy Relations}

\subsection{Common Common-Cause}

Before discussing the possibility of separate common cause model, in this section, I will show the non-existence of the local common common-cause model. After that, in Sect. 3.2, referring to the proof method in this section, I will show the non-existence of a local separate common cause model similarly. Comparing these proofs will reveal whether the strategy of weakening the requirement for common common-cause to the requirement for a separate common-cause is effective or not.

As with many textbooks (e.g. $[1,2])$, we require the following as the conditions that the local common common-cause model should satisfy.

C1 There exist common screening-off factors $\left\{C_{k}\right\}_{k \in K}$ for the correlations $\operatorname{Corr}\left(L_{1+}, R_{2+}\right)$ and $\operatorname{Corr}\left(L_{2+}, R_{1+}\right){ }^{3}$

C2-1 $\operatorname{Pr}\left(L_{i+} \mid L_{i} R_{j} C_{k}\right)=\operatorname{Pr}\left(L_{i+} \mid L_{i} R_{j^{\prime}} C_{k}\right)$.

C2-2 $\operatorname{Pr}\left(R_{j+} \mid L_{i} R_{j} C_{k}\right)=\operatorname{Pr}\left(R_{j+} \mid L_{i^{\prime}} R_{j} C_{k}\right)$.

\footnotetext{
${ }^{3}\left\{C_{k}\right\}_{k \in K}$ is common screening-off factors for the two correlations, so the superscript of screening-off factors in Definition 1 is omitted.
} 
C3 $\operatorname{Pr}\left(C_{k} \mid L_{i} R_{j}\right)=\operatorname{Pr}\left(C_{k}\right)$.

$\mathrm{C} 2$ requires that when the cause of the correlation occurs, the measurement result of one system does not depend on the setting of the measurement apparatus of the other system. Taking $\mathrm{C} 2-1$ as an example, this condition requires that $\operatorname{Pr}\left(L_{1+} \mid L_{1} R_{2} C_{k}\right)=\operatorname{Pr}\left(L_{1+} \mid L_{1} R_{1} C_{k}\right)$ for the correlation $\operatorname{Corr}\left(L_{1+}, R_{2+}\right)$ and that $\operatorname{Pr}\left(L_{2+} \mid L_{2} R_{1} C_{k}\right)=\operatorname{Pr}\left(L_{2+} \mid L_{2} R_{2} C_{k}\right)$ for the correlation $\operatorname{Corr}\left(L_{2+}, R_{1+}\right)$.

C3 requires that settings of the measurement apparatus are independent of common causes. It is natural to think that the common-cause event of correlation between the events occurring on two spatially separated regions occurs in the common past of those two regions. Considering that the settings of the apparatus can be changed even after the events that occurred in the common past have been completely specified, C3 is a requirement that should be met.

I show that there exists no classical probability space that is consistent with Hardy relations B1-B4 and that satisfies C1-C3.

Proposition 1. Suppose that there exist common screening-off factors $\left\{C_{k}\right\}_{k \in K}$ of the correlations $\operatorname{Corr}\left(L_{1}, R_{2+}\right)$ and $\operatorname{Corr}\left(L_{2+}, R_{1+}\right)$ and that $C_{2}$ and $C 3$ are satisfied for any $C_{k}$. Then, a contradiction with Hardy relations B1-B4 is derived.

Proof. By (a), (b), and (c) of Definition 1,

$$
\operatorname{Pr}\left(L_{i a} R_{j b} \mid L_{i} R_{j}\right)=\sum_{k} \operatorname{Pr}\left(L_{i a} R_{j b} \mid L_{i} R_{j} C_{k}\right) \cdot \operatorname{Pr}\left(C_{k} \mid L_{i} R_{j}\right) .
$$

holds for any $i, j$ and $a, b$. Using C3, we have

$$
\operatorname{Pr}\left(L_{i a} R_{j b} \mid L_{i} R_{j}\right)=\sum_{k} \operatorname{Pr}\left(L_{i a} R_{j b} \mid L_{i} R_{j} C_{k}\right) \cdot \operatorname{Pr}\left(C_{k}\right) .
$$

Then, from (1) and B1, there exists $k^{\prime}$ such that

$$
\operatorname{Pr}\left(L_{1+} R_{1+} \mid L_{1} R_{1} C_{k^{\prime}}\right)>0 .
$$

By B2, either $\operatorname{Pr}\left(R_{2+} \mid L_{1} R_{2} L_{1+} C_{k^{\prime}}\right)=1$ or $\operatorname{Pr}\left(L_{1} R_{2} L_{1+} C_{k^{\prime}}\right)=0$ holds. However, as we will see, we get $\operatorname{Pr}\left(L_{1} R_{2} L_{1+} C_{k^{\prime}}\right) \neq 0$. Using C2-1 we have

$$
\begin{aligned}
\operatorname{Pr}\left(L_{1+} R_{1+} \mid L_{1} R_{1} C_{k^{\prime}}\right) & \leq \operatorname{Pr}\left(L_{1+} \mid L_{1} R_{1} C_{k^{\prime}}\right) \\
& =\operatorname{Pr}\left(L_{1+} \mid L_{1} R_{2} C_{k^{\prime}}\right) .
\end{aligned}
$$

From this and (2), we get $\operatorname{Pr}\left(L_{1+} \mid L_{1} R_{2} C_{k^{\prime}}\right)>0$, then we have $\operatorname{Pr}\left(L_{1} R_{2} L_{1+} C_{k^{\prime}}\right) \neq$ 0 . Therefore,

$$
\operatorname{Pr}\left(R_{2+} \mid L_{1} R_{2} L_{1+} C_{k^{\prime}}\right)=1 .
$$

From Fact 1 (i) and C2-2, we get

$$
\begin{aligned}
\operatorname{Pr}\left(R_{2+} \mid L_{1} R_{2} L_{1+} C_{k^{\prime}}\right) & =\operatorname{Pr}\left(R_{2+} \mid L_{1} R_{2} C_{k^{\prime}}\right) \\
& =\operatorname{Pr}\left(R_{2+} \mid L_{2} R_{2} C_{k^{\prime}}\right) .
\end{aligned}
$$


From this and (3),

$$
\operatorname{Pr}\left(R_{2+} \mid L_{2} R_{2} C_{k^{\prime}}\right)=1
$$

Also, starting with B3, as a result of the same derivation as in the previous paragraph, we get

$$
\operatorname{Pr}\left(L_{2+} \mid L_{2} R_{2} C_{k^{\prime}}\right)=1
$$

Then, from (4) and (5)

$$
\operatorname{Pr}\left(L_{2+} R_{2+} \mid L_{2} R_{2} C_{k^{\prime}}\right)=1,
$$

therefore, by (1),

$$
\operatorname{Pr}\left(L_{2+} R_{2+} \mid L_{2} R_{2}\right)>0 .
$$

However, (7) contradicts with B4.

As mentioned above, when a two-particle system is in a maximally entangled state, there exist four projections satisfying Hardy relations. When a pair of particles is in such a state, by Proposition 1, there exists no local common common-cause model consistent with quantum mechanical predictions.

\subsection{Separate Common-Cause}

Higashi [14] showed that there exists no local separate common cause model consistent with Hardy relations. In this section, I will give another simple proof of the same content. The proof proceeds similarly to the no-go proof for common common-causes in Sect. 3.1.

As in Higashi [14], we require the following conditions which the local separate common-cause model should satisfy.

S1 There exist screening-off factors $\left\{C_{k}^{12}\right\}_{k \in K}$ and $\left\{C_{l}^{21}\right\}_{l \in L}$ for the correlations $\operatorname{Corr}\left(L_{1+}, R_{2+}\right)$ and $\operatorname{Corr}\left(L_{2+}, R_{1+}\right)$ respectively.

S2-1 $\operatorname{Pr}\left(L_{i+} \mid L_{i} R_{j} C_{m}^{i j}\right)=\operatorname{Pr}\left(L_{i+} \mid L_{i} R_{j^{\prime}} C_{m}^{i j}\right)$.

S2-2 $\operatorname{Pr}\left(R_{j+} \mid L_{i} R_{j} C_{m}^{i j}\right)=\operatorname{Pr}\left(R_{j+} \mid L_{i^{\prime}} R_{j} C_{m}^{i j}\right)$.

S3 Let $\mathcal{C}$ be the Boolean subalgebra generated by $\left\{C_{k}^{12}\right\}_{k \in K} \cup\left\{C_{l}^{21}\right\}_{l \in L}$. Then, for any element $Z$ of $\mathcal{C}$,

$$
\operatorname{Pr}\left(Z \mid L_{i} \wedge R_{j}\right)=\operatorname{Pr}(Z) \quad(i, j=1,2) .
$$

$\mathrm{S} 2$ is a rewrite of the condition $\mathrm{C} 2$ for the common common-cause model to a condition for the separate common-cause model. Taking S2-1 as an example, the condition requires that $\operatorname{Pr}\left(L_{1+} \mid L_{1} R_{2} C_{k}^{12}\right)=\operatorname{Pr}\left(L_{1+} \mid L_{1} R_{1} C_{k}^{12}\right)$ for the correlation $\operatorname{Corr}\left(L_{1+}, R_{2+}\right)$ and that $\operatorname{Pr}\left(L_{2+} \mid L_{2} R_{1} C_{l}^{21}\right)=\operatorname{Pr}\left(L_{1+} \mid L_{2} R_{2} C_{l}^{21}\right)$ for the correlation $\operatorname{Corr}\left(L_{2+}, R_{1+}\right)$.

S3 requires that apparatus settings are completely independent of the subalgebra of common causes of various correlations. ${ }^{4}$ As stated for the condition

\footnotetext{
${ }^{4}$ In Higashi [14], this requirement is stated in a different form (called $\mathcal{C}$-independence) however, they are mathematically equivalent.
} 
C3 for the common common-cause, it is natural to think that common cause events occur in the common past, and furthermore we can change the measurement settings even after the events in the common past have been completely specified. Then, S3 is a requirement to be satisfied.

I show that there exists no classical probability space that is consistent with Hardy relations B1-B4 and that satisfies S1-S3.

Proposition 2. Suppose that there exist screening-off factors $\left\{C_{k}^{12}\right\}_{k \in K}$ and $\left\{C_{l}^{21}\right\}_{l \in L}$ for the correlations $\operatorname{Corr}\left(L_{1+}, R_{2+}\right)$ and $\operatorname{Corr}\left(L_{2+}, R_{1+}\right)$ respectively and that S2 and $S 3$ are satisfied for any $C_{k}^{12}$ and $C_{l}^{21}$. Then, a contradiction with Hardy relations $B 1-B 4$ is derived.

Proof. Using the screening-off factors $\left\{C_{k}^{12}\right\}_{k \in K}$ and $\left\{C_{l}^{21}\right\}_{l \in L}$, we define the following set of events:

$$
\mathcal{S} \equiv\left\{C_{k}^{12} C_{l}^{21}: k \in K, l \in L, P r\left(C_{k}^{12} C_{l}^{21}\right)>0\right\} .
$$

Clearly, by Definition 1 and the definition of $\mathcal{S}$

$$
\operatorname{Pr}\left(L_{i a} R_{j b} \mid L_{i} R_{j}\right)=\sum_{\mathcal{S}} \operatorname{Pr}\left(L_{i a} R_{j b} \mid L_{i} R_{j} C_{k}^{12} C_{l}^{21}\right) \cdot \operatorname{Pr}\left(C_{k}^{12} C_{l}^{21} \mid L_{i} R_{j}\right)
$$

where, $\sum_{\mathcal{S}}$ denotes the summation of the value of $\operatorname{Pr}\left(L_{i a} R_{j b} \mid L_{i} R_{j} C_{k}^{12} C_{l}^{21}\right)$. $\operatorname{Pr}\left(C_{k}^{12} C_{l}^{21} \mid L_{i} R_{j}\right)$ over all the events belonging to the set $\mathcal{S}$.

Using S3, we have

$$
\operatorname{Pr}\left(L_{i a} R_{j b} \mid L_{i} R_{j}\right)=\sum_{k, l \in \mathcal{S}} \operatorname{Pr}\left(L_{i a} R_{j b} \mid L_{i} R_{j} C_{k}^{12} C_{l}^{21}\right) \cdot \operatorname{Pr}\left(C_{k}^{12} C_{l}^{21}\right) .
$$

Then, from (8) and Hardy relation B1, for some $k^{\prime}$ and $l^{\prime}$

$$
\operatorname{Pr}\left(L_{1+} R_{1+} \mid L_{1} R_{1} C_{k^{\prime}}^{12} C_{l^{\prime}}^{21}\right)>0 .
$$

From the inequality (9), we have

$$
\operatorname{Pr}\left(L_{1+} R_{1+} \mid L_{1} R_{1} C_{k^{\prime}}^{12}\right)>0 .
$$

In the proof of Proposition 1, using B2 and C2, we obtained (4) from (2). Using $\mathrm{S} 2$ instead of $\mathrm{C} 2$, as a result of the same derivation, from (10) we get

$$
\operatorname{Pr}\left(R_{2+} \mid L_{2} R_{2} C_{k^{\prime}}^{12}\right)=1
$$

From (9), we can get also

$$
\operatorname{Pr}\left(L_{1+} R_{1+} \mid L_{1} R_{1} C_{l^{\prime}}^{21}\right)>0 .
$$

Then, for the same reason as stated in the previous paragraph, using B3 and $\mathrm{C} 2$, we get

$$
\operatorname{Pr}\left(L_{2+} \mid L_{2} R_{2} C_{l^{\prime}}^{21}\right)=1 \text {. }
$$


From (11) and (12) we have

$$
\operatorname{Pr}\left(L_{2+} R_{2+} \mid L_{2} R_{2} C_{k^{\prime}}^{12} C_{l^{\prime}}^{21}\right)=1 .
$$

Then, by (8) and (13),

$$
\operatorname{Pr}\left(L_{2+} R_{2+} \mid L_{2} R_{2}\right)>0 .
$$

However, (14) contradicts B4.

When a pair of particles is in a maximally entangled state, by Proposition 2 , there exists no local separate common-cause model consistent with quantum mechanical predictions.

\subsection{Summary}

In Proposition 1, I showed that there exists no local common common-cause model of Hardy relations. If such a model exists, there must exist an event that satisfies (2) among the common screening-off factors. When there exists at least one event that satisfying (2), a contradiction with B4 is derived. In Proposition 2, I showed that there exists no local separate common-cause model of Hardy relations. The proof-method of Proposition 2 was almost the same as when Proposition 1 was shown. We can construct an event satisfying (9) from the screening-off factors of each correlation. When at least one event satisfying (9) exists, a contradiction with B4 is derived.

\section{Reducible or Irreducible?}

\subsection{The Issue of Reducibility}

As stated in Sect. 1, there are various no-go theorems that showed the nonexistence of the separate common-cause model. In the papers except for Higashi [14] and this paper, it was showed that there exists no local common-cause model for the correlations that appear in Bell-type inequalities (Bell correlations). One of the most influential of those papers is that of Graßhoff et al. [7]. They showed the non-existence of a local separate common-cause model in situations where various combinations of spin quantities, including three pairs with perfect (anti)correlation, could be measured (e.g. the situation where various combinations of spin quantities including three parallel settings are measurable for a pair of spin $1 / 2$ particles in the singlet state). With respect to the proof given by Graßhoff et al. Hofer-Szabó [10] analyzed that although their proof does not presuppose the existence of common screening-off factors for multiple correlations, in fact, their assumptions for the separate common-cause model imply the existence of the common common-cause model. More specifically, in the particular situation they used to prove (i.e. the singlet state and three parallel settings), if for each correlation, there exist separate screening factors satisfying S2 and S3, we can construct common screening-off factors satisfying C2 and C3. 
First, as far as necessary in the following discussion, let us review HoferSzabó's analysis. Suppose that there exist screening-off factors $\left\{C_{j_{i i}}^{i i}\right\}_{j_{i i} \in J_{i i}}(i=$ $1,2,3)$ of three pairs of perfect (anti-)correlations $\operatorname{Corr}\left(L_{i a}, R_{i b}\right)(i=1,2,3)$, respectively, which satisfy $\mathrm{S} 2$ and $\mathrm{S} 3$. As is well known (e.g. [20]), when there is a perfect (anti) correlation, the determinism in the following sense holds for the conditional probability given any event belonging to the screening-off factors.

$$
\operatorname{Pr}\left(L_{i a} \mid L_{i} R_{i} C_{j_{i i}}^{i i}\right)=1 \text { or } 0 ; \operatorname{Pr}\left(R_{i b} \mid L_{i} R_{i} C_{j_{i i}}^{i i}\right)=1 \text { or } 0 .
$$

This is a non-local determinism in which the probability of the measurement result for one system depends on the apparatus setting for the other system, however, using S2, we get the following local determinism:

$$
\operatorname{Pr}\left(L_{i a} \mid L_{i} C_{j_{i i}}^{i i}\right)=1 \text { or } 0 ; \operatorname{Pr}\left(R_{i b} \mid R_{i} C_{j_{i i}}^{i i}\right)=1 \text { or } 0 .
$$

In general, for any two events $E$ and $F$, if $\operatorname{Pr}(F \mid E)=1(0)$ holds, then $\operatorname{Pr}(F \mid E G)=1(0)$ holds for any event $G$ such that $\operatorname{Pr}(E G) \neq 0$. Thus, for any event $C_{j_{11}}^{11} C_{j_{22}}^{22} C_{j_{33}}^{33}$ constructed from the screening-off factors of each correlation, if $\operatorname{Pr}\left(C_{j_{11}}^{11} C_{j_{22}}^{22} C_{j_{33}}^{33}\right) \neq 0$, then we get

$$
\operatorname{Pr}\left(L_{i a} \mid L_{i} C_{j_{11}}^{11} C_{j_{22}}^{22} C_{j_{33}}^{33}\right)=1 \text { or } 0 ; \operatorname{Pr}\left(R_{i b} \mid R_{i} C_{j_{11}}^{11} C_{j_{22}}^{22} C_{j_{33}}^{33}\right)=1 \text { or } 0 .
$$

When this form of determinism holds, we can easily prove that the events

$$
\mathcal{T} \equiv\left\{C_{j_{11}}^{11} C_{j_{22}}^{22} C_{j_{33}}^{33}: j_{11} \in J_{11}, j_{22} \in J_{22}, j_{33} \in J_{33}, \operatorname{Pr}\left(C_{j_{11}}^{11} C_{j_{22}}^{22} C_{j_{33}}^{33}\right) \neq 0\right\}
$$

are common screening-off factors of all correlations and that they satisfy $\mathrm{C} 2$. (Note that C3 is immediately derived from S3.) Then, for example, $\operatorname{Pr}\left(L_{1+} R_{2+}\right.$ । $\left.L_{1} R_{2}\right)$ is factorized as follows:

$$
\operatorname{Pr}\left(L_{1+} R_{2+} \mid L_{1} R_{2}\right)=\sum_{i \in I} \operatorname{Pr}\left(L_{1+} \mid L_{1} C_{i}\right) \operatorname{Pr}\left(R_{2+} \mid R_{2} C_{i}\right) \operatorname{Pr}\left(C_{i}\right)
$$

(where $\mathcal{T}$ was rewritten as $\mathcal{T}=\left\{C_{i}\right\}_{i \in I}$ ). After that, Bell-type inequalities are derived in a well-known way.

In short, as Hofer-Szabó [10] correctly pointed out, in the particular situation where a pair of particles in a maximally entangled state are measured under settings including three parallel settings, the non-existence proof of the local separate common-cause model is reduced to the non-existence proof of the local common common-cause model. In this situation, the existence of a local separate common-cause model implies the existence of a local common common-cause model. Therefore, there is no practical meaning in weakening a requirement for a common common-cause to a separate common-cause.

By the way, is the non-existence proof of the local separate common-cause model that satisfies Hardy relations also reduced to the proof of the nonexistence of the local common common-cause model similarly? If "reduction" is attempted in the same way as in the case of Bell correlations, the following two points need to be discussed. 
1. As confirmed above, in the case of Bell correlations, the reduction of nonexistence proof required a logical partition, ${ }^{5}$ i.e. common screening-off factors for multiple correlations (see $\mathcal{T}$ and the equation (15)). On the other hand, Proposition 1 and 2 respectively hold even if there exists only one event satisfying the inequality (2) and (9).

2. In the case of Bell correlations, the non-existence proof was given in the specified situation of three (anti-)perfect correlations. As stated above, in this situation, determinism holds, and so we can construct common screening-off factors satisfying $\mathrm{C} 2$ and $\mathrm{C} 3$. Then, are the correlations in Hardy relations perfect correlations or not?

We will discuss each of these issues below.

\subsection{A single event or logical partition?}

When there exists at least one event satisfying the inequalities (2) and (9) respectively, we can show Proposition 1 and 2. Therefore, I initially thought that in the case of Hardy relations, unlike Bell correlation, which uses logical partitions for the proof, it is meaningless to discuss the problem of reducibility. However now I think that idea is too naive. There are two reasons.

First, if it is possible to construct common screening-off factors satisfying $\mathrm{C} 2$ and $\mathrm{C} 3$ from separate screening-off factors of each correlation that satisfy S2 and S3, then by applying Proposition 1 to those events, we can show the non-existence of the local separate common-cause model. Such a proof is a little detour however, if truly "reducible", nothing is mathematically incorrect.

Second, there is another proof that uses a logical partition of events (rather than a single event) to show the non-existence of the local common-cause model satisfying Hardy relations. I explain the proof method. The following fact holds.

Fact 2. Suppose there exist common screening-off factors $\left\{C_{k}\right\}_{k \in K}$ of the correlations $\operatorname{Corr}\left(L_{1+}, R_{2+}\right)$ and $\operatorname{Corr}\left(L_{2+}, R_{1+}\right)$ and furthermore that C2 and C3 hold. Then, for any $C_{k}$,

$$
\operatorname{Pr}\left(L_{1+} R_{1+} \mid L_{1} R_{1} C_{k}\right) \leq \operatorname{Pr}\left(L_{2+} R_{2+} \mid L_{2} R_{2} C_{k}\right) .
$$

Proof. When the value on the left side is $0,(16)$ clearly holds. Therefore, it is sufficient to show (16) holds when $\operatorname{Pr}\left(L_{1+} R_{1+} \mid L_{1} R_{1} C_{k}\right)>0$. If $\operatorname{Pr}\left(L_{1+} R_{1+} \mid\right.$ $\left.L_{1} R_{1} C_{k}\right)>0$, then we get $\operatorname{Pr}\left(L_{2+} R_{2+} \mid L_{2} R_{2} C_{k}\right)=1$ in the same way that we derived (6) from (2) in the proof of Proposition 1. Then, (16) clearly holds.

By Definition 1 and C3, for any $i(i=1,2)$

$$
\operatorname{Pr}\left(L_{i+} R_{i+} \mid L_{i} R_{i}\right)=\sum_{k \in K} \operatorname{Pr}\left(L_{i+} R_{i+} \mid L_{i} R_{i} C_{k}\right) \cdot \operatorname{Pr}\left(C_{k}\right) .
$$

\footnotetext{
${ }^{5}$ To be exact, the screening-off factors are not always a logical partition. However, even in such a case, if one event having probability 0 is added, the logical partition is obtained.
} 
Then, from Fact 2, we have

$$
\operatorname{Pr}\left(L_{1+} R_{1+} \mid L_{1} R_{1}\right) \leq \operatorname{Pr}\left(L_{2+} R_{2+} \mid L_{2} R_{2}\right)
$$

and so by B1, $0<\operatorname{Pr}\left(L_{2+} R_{2+} \mid L_{2} R_{2}\right)$. That contradicts B4.

Suppose there exist separate screening-off factors $\left\{C_{k}^{12}\right\}_{k \in K}$ and $\left\{C_{l}^{21}\right\}_{l \in L}$ for the correlations $\operatorname{Corr}\left(L_{1+}, R_{2+}\right)$ and $\operatorname{Corr}\left(L_{2+}, R_{1+}\right)$, respectively, and those events meet S2 and S3. If we can construct the common screening-off factors of $\operatorname{Corr}\left(L_{1+}, R_{2+}\right)$ and $\operatorname{Corr}\left(L_{2+}, R_{1+}\right)$ from the separate ones $\left(\left\{C_{k}^{12}\right\}_{k \in K}\right.$ and $\left.\left\{C_{l}^{21}\right\}_{l \in L}\right)$, and furthermore if those events satisfy C2 and C3, then using Fact 2, we can show the non-existence of local separate common-cause model. In short, the non-existence proof of the local separate common-cause model is reduced to the non-existence proof of the local common common-cause model. In this proof, the probabilities of each event in the logical partition are summed, as in the case of Bell correlation. Even in the case of Hardy relations, It is not meaningless to discuss the issue of reducibility.

\subsection{Perfect Correlation or not?}

In this section, let us consider whether the correlations $\operatorname{Corr}\left(L_{1+}, R_{2+}\right)$ and $\operatorname{Corr}\left(L_{2+}, R_{1+}\right)$ in Hardy relations are perfect correlations or not. Because, as mentioned above, if these are perfect correlations, then determinism holds, and so common screening-off factors of those correlations can be constructed. However, we can show that $\operatorname{Corr}\left(L_{1+}, R_{2+}\right)$ and $\operatorname{Corr}\left(L_{2+}, R_{1+}\right)$ are not perfect correlations.

Fact 3. In a pure state $\phi$ in $\mathcal{H}_{L} \otimes \mathcal{H}_{R}$, we assume that Hardy relations (17)-(20) hold and that the correlation $\operatorname{Corr}\left(L_{1+}, R_{2+}\right)$ is a perfect correlation (i.e. not only (18) but also (21) holds). Then, (18)-(21) exclude (17) and so a contradiction is derived. For $\operatorname{Corr}\left(L_{2+}, R_{1+}\right)$, similarly, a contradiction is derived.

Proof. The following relations holds for the pure state $\phi$ in $\mathcal{H}_{L} \otimes \mathcal{H}_{R}$.

$$
\begin{aligned}
Q^{\phi}\left(A_{L} A_{R}\right) & >0, \\
Q^{\phi}\left(B_{R} \mid A_{L}\right) & =1, \\
Q^{\phi}\left(B_{L} \mid A_{R}\right) & =1, \\
Q^{\phi}\left(B_{L} B_{R}\right) & =0, \\
Q^{\phi}\left(A_{L} \mid B_{R}\right) & =1 .
\end{aligned}
$$

From (18) and (21), we get respectively

$$
\begin{aligned}
& B_{R} A_{L} \phi=A_{L} \phi, \\
& A_{L} B_{R} \phi=B_{R} \phi .
\end{aligned}
$$

$A_{L}$ and $B_{R}$ are commutative, and so from (22) and (23), we get

$$
A_{L} \phi=B_{R} \phi
$$


From (20),

$$
B_{L} B_{R} \phi=\mathbf{0},
$$

where $\mathbf{0}$ is the zero vector. Then, from (24) and (25), $B_{L} A_{L} \phi=B_{L} B_{R} \phi=\mathbf{0}$, and so we have

$$
B_{L} A_{L} \phi=\mathbf{0} .
$$

Using (26), $B_{L} A_{L}^{\perp} \phi=B_{L} \phi-B_{L} A_{L} \phi=B_{L} \phi$ (where $A_{L}^{\perp}=I-A_{L}$ ) and so

$$
B_{L} A_{L}^{\perp} \phi=B_{L} \phi .
$$

Using (27) and the commutativity of operators of the left and right system, $B_{L} A_{R} \phi=A_{R} B_{L} \phi=A_{R} B_{L} A_{L}^{\perp} \phi=B_{L} A_{L}^{\perp} A_{R} \phi$, and so we have

$$
B_{L} A_{R} \phi=B_{L} A_{L}^{\perp} A_{R} \phi .
$$

Also,

$$
\left\|B_{L} A_{L}^{\perp} A_{R} \phi\right\| \leq\left\|A_{L}^{\perp} A_{R} \phi\right\| .
$$

Therefore, from (28) and (29), we have $\left\|B_{L} A_{R} \phi\right\| \leq\left\|A_{L}^{\perp} A_{R} \phi\right\|$, and so

$$
\frac{\left\|B_{L} A_{R} \phi\right\|^{2}}{\left\|A_{R} \phi\right\|^{2}} \leq \frac{\left\|A_{L}^{\perp} A_{R} \phi\right\|^{2}}{\left\|A_{R} \phi\right\|^{2}} .
$$

From (19), the left side of (30) is 1, and so the right side also is 1, therefore we get $Q^{\phi}\left(A_{L}^{\perp} \mid A_{R}\right)=1$. Then,

$$
Q^{\phi}\left(A_{L} A_{R}\right)=0
$$

and this contradicts (17).

Note two things about this proof.

- Hardy actually constructed projection operators satisfying (17) through (20). Fact 3 holds for arbitrary projections satisfying (17)-(20), not just the actually constructed projections.

- As Hardy himself pointed out, Hardy relations only hold for non-maximally entangled states. However, proof of Fact 3 did not use the distinction between maximally and non-maximally.

From Fact 3 , the correlations $\operatorname{Corr}\left(L_{1+}, R_{2+}\right)$ and $\operatorname{Corr}\left(L_{2+}, R_{1+}\right)$ in Hardy Relations cannot be perfect correlations. Therefore, determinism cannot be derived. Then, we cannot construct common screening-off factors satisfying $\mathrm{C} 2$ and C3 in the way described in Sect. 4.1. 


\subsection{Concluding Remark}

As we saw in Sect. 4.2, we can rewrite the proof of Proposition 1 into a form that uses logical partition instead of a single event, just as in the case of Bell correlations. However, as stated in Sect. 4.3, we can not derive determinism. Thus, in the way described in Sect. 4.1, we can not construct common screeningoff factors, and even if we can, those events do not necessarily satisfy $\mathrm{C} 2$ and C3.

On the other hand, the following is also true. I have only confirmed that a particular method constructing common screening-off factors satisfying $\mathrm{C} 2$ and C3 from the separate screening-off factors satisfying S2 and S3 does not work. However, it may be possible to extend the algebra of separate screening-off factors to algebra including common screening-off factors satisfying C2 and C3 (e.g. the method Wroński et al. used for Bell-Aspect correlations, ${ }^{6}$ see Sect. 5.6 in [13]). To show irreducibility rigorously, not only we confirm that a particular method does not work, but also we need to show that, no matter what logical partition is constructed, there exist no common screening-off factors satisfying $\mathrm{C} 2$ and $\mathrm{C} 3$. This paper does not answer the issue of reducibility in this strict sense, and I do not currently know the answer to it.

\section{References}

[1] Redhead, M.: Incompleteness, Nonlocality, and Realism. Oxford University Press, Oxford (1987)

[2] Bub, J.: Interpreting the Quantum World. Cambridge University Press, Cambridge (1997)

[3] Aspect, A., Dalibard, J., Roger, G.: Experimental test of Bell's inequalities using time-varying analyzers. Phys. Rev. Lett. 49, 1804-1807 (1982)

[4] Hofer-Szabó, G., Rédei, M., Szabó, L. E.: On Reichenbach's common cause principle and Reichenbach's notion of common cause. Br. J. Philos. Sci. 50, 377-399 (1999)

[5] Szabó, L. E.: Attempt to resolve the EPR-Bell paradox via Reichenbach's concept of common cause. Int. J. Theor. Phys. 39, 901-911 (2000)

[6] Hofer-Szabó, G., Rédei, M., Szabó, L. E.: Common-causes are not common common-causes. Philos. Sci. 69, 623-636 (2002)

[7] Graßhoff, G., Portmann, S., Wüthrich, A.: Minimal assumption derivation of a Bell-type inequality. Br. J. Philos. Sci. 56, 663-680 (2005)

[8] Portmann, S., Wüthrich, A.: Minimal assumption derivation of a weak Clauser-Horne inequality. Stud. Hist. Philos. Sci. B 38, 844-862 (2007)

\footnotetext{
${ }^{6}$ However, as they themselves know, they assume a stronger condition than S2.
} 
[9] Higashi, K.: The limits of common cause approach to EPR correlation. Found. Phys. 38, 591-609 (2008)

[10] Hofer-Szabó, G.: Separate-versus common-common-cause-type derivations of the Bell inequalities. Synthese 163, 199-215 (2008)

[11] Hofer-Szabó, G.: Bell $(\delta)$ inequalities derived from separate common causal explanation of almost perfect EPR anticorrelations. Found. Phys. 41, 13981413 (2011)

[12] Hofer-Szabó, G.: Separate common causal explanation and the Bell inequalities. Int. J. Theor. Phys. 51, 110-123 (2012)

[13] Wroński, L., Placek, T., Godziszewski, M. T.: Separate common causes and EPR correlations - an "almost no-go" result. In: Hofer-Szabó, G., Wroński, L. (eds.) Making It Formally Explicit, pp. 85-107. Springer, Cham (2017)

[14] Higashi, K.: A no-go result on common cause approaches via Hardy relations. Stud. Hist. Philos. Sci. B 67, 12-19 (2019)

[15] Nielsen, M., Chuang, I.: Quantum Computation and Quantum Information. Cambridge University Press, Cambridge (2000)

[16] Hardy, L.: Nonlocality for two particles without inequalities for almost all entangled states. Phys. Rev. Lett. 71, 1665-1668 (1993)

[17] Szabó, L. E.: Is quantum mechanics compatible with a deterministic universe? Two interpretations of quantum probabilities. Found. Phys. Lett. 8, 421-440 (1995)

[18] Bana, G., Durt, T.: Proof of Kolmogorovian censorship. Found. Phys. 27, $1355-1373$ (1997)

[19] Reichenbach, H.: The Direction of Time. Dover Publications, New York (1956)

[20] Van Fraassen, B. C.: The charybdis of realism: epistemological implications of Bell's inequality. Synthese 52, 25-38 (1982) 